\title{
LA COLABORACIÓN Y LA FORMACIÓN DEL PROFESORADO COMO FACTORES FUNDAMENTALES PARA PROMOVER UNA EDUCACIÓN SIN EXCLUSIONES
}

\author{
Marta Sandoval Mena \\ Universidad Autónoma de Madrid
}

\section{RESUMEN}

Una de las palancas que mueven la escuela inclusiva es la colaboración como modo de trabajo del profesorado, hecho que se constata en numerosas evidencias de investigación para gestionar la diversidad del aula en colaboración con otros agentes: profesores, padres, alumnos, etc. En este artículo se analizan las barreras que impiden o dificultan la colaboración entre el profesorado con el fin de impulsar el análisis de la formación docente actual como elemento clave para avanzar hacia una educación inclusiva.

Palabras clave: Inclusión educativa, colaboración, profesor de apoyo, formación docente

\section{ABSTRACT}

The cooperation understood as teachers ' way of work is one of the levers that move on the inclusive education. In this article we try to rethink the education training as a key in order to progress inclusive education. It shows research evidences to attend the diversity into classrooms and it analyses some barriers on working variety among educators.

With this work, our intention is promoting a working line only slightly established in our professional practices: collaborative work.

Key words: Educational inclusion, cooperation, Special Education Needs teacher, teaching training. 


\section{La palanca hacia la inclusión: la colaboración}

La inclusión educativa impregna cambios sustantivos en los contextos físicos, curriculares y organizativos en las escuelas y promueve la transformación de las políticas y prácticas educativas de los centros para avanzar hacia una educación de calidad para todos los alumnos.

Desde la evidencia teórica y empírica que disponemos, este tipo de cambios se producen fundamentalmente movidos por unas condiciones concretas, tanto del centro como externas al mismo, y a través de unos determinados valores y expectativas, que se hacen realidad a través de procesos de colaboración y participación de toda la comunidad escolar. Será a través de estos procesos de colaboración los que nos impulsen a replantear las actuales prácticas docentes en este artículo.

A pesar de la variedad de experiencias de inclusión entre unos contextos y otros, se podría hablar de unas condiciones necesarias para desarrollar un modelo inclusivo de educación. A continuación, se presentan algunas de las condiciones recogidas por diferentes autores (ver tabla I), que ponen de manifiesto el papel tan relevante que tiene el profesor como colaborador y responsable de todos los alumnos de la clase.

Por tanto, cuando se habla de inclusión educativa, siempre está presente la figura del profesor como un profesional que no puede entender su trabajo sin la colaboración con otros profesionales y agentes educativos tanto del centro como externos al mismo; es más, se percibe el aislamiento profesional en este ámbito como una de las principales dificultades que interfieren en la atención a la diversidad. La colaboración se convierte, en definitiva, en una palanca decisiva para construir "una escuela para todos".

Resulta ingenuo, por no decir imposible, que el profesor pueda por sí sólo dar respuesta a las múltiples exigencias que se le presentan y atender a la diversidad de competencias, intereses y necesidades del aula. Si pensamos en una práctica profesional sin esta condición, no puede extrañarnos que el profesorado dude de sus capacidades para hacer frente a las demandas del alumnado y compatibilizar, además, estas exigencias con las de la propia administración. La empresa, es demasiado ardua si trabajamos solos, las funciones se han multiplicado y son necesarios apoyos (emocionales, didácticos, tecnológicos...) para hacer frente a todas ellas.

La colaboración es un entramado complejo en el que entran en juego un conjunto de valores, creencias, normas, destrezas, patrones de acción, roles modelos de relación, etc. que los docentes comparten en un contexto de aprendizaje interactivo. En nuestros centros, la colaboración tiene multitud de formas. Necesitamos avanzar en los diferentes tipos de colaboración como son los proyectos de cooperación entre centros escolares, entre profesionales, con las familias, con los servicios educativos y/o sociales del entorno, etc.. Según Parrilla (2007) estamos ante una de las tareas pendientes y necesarias para favorecer cualquier proceso inclusivo: 
Tabla 1. Condiciones para la inclusión educativa propuestas por diferentes autores

Porter (2005)

Ainscow (1995)

Moriña y

Parrilla (2005)

\begin{tabular}{l}
\hline Estrategias \\
educativas \\
múltiples en una \\
clase \\
("enseñanza a \\
niveles \\
múltiples").
\end{tabular}

Liderazgo

consensuado y pedagógico.

compartido.

\section{Arnaiz (2003) Echeita (2006)}

tendrían que

comportar como

Comunidades de

aprendizaje.

\author{
Profesional \\ reflexivo, crítico, \\ estratégico.
}

\begin{tabular}{|c|c|c|c|c|}
\hline $\begin{array}{l}\text { Rol de los } \\
\text { profesores de } \\
\text { apoyo más } \\
\text { amplio del } \\
\text { tradicional sus } \\
\text { funciones se } \\
\text { dirigen a todos los } \\
\text { alumnos que lo } \\
\text { necesiten. }\end{array}$ & $\begin{array}{l}\text { Compromiso con } \\
\text { toda la } \\
\text { comunidad } \\
\text { educativa, } \\
\text { creando } \\
\text { actividades para } \\
\text { implicar a } \\
\text { alumnos, padres y } \\
\text { profesores. }\end{array}$ & $\begin{array}{l}\text { Implicación de la } \\
\text { comunidad } \\
\text { educativa. }\end{array}$ & $\begin{array}{l}\text { Profesor reflexivo, } \\
\text { consciente de que } \\
\text { más que un } \\
\text { técnico es un } \\
\text { intelectual } \\
\text { consciente de las } \\
\text { dimensiones } \\
\text { éticas de la } \\
\text { profesión. }\end{array}$ & $\begin{array}{l}\text { Profesionales } \\
\text { comprometidos } \\
\text { que valoren y } \\
\text { asuman la } \\
\text { responsabilidad } \\
\text { de todos los } \\
\text { alumnos del aula }\end{array}$ \\
\hline
\end{tabular}

\begin{tabular}{|c|c|c|c|c|}
\hline $\begin{array}{l}\text { Desarrollo de } \\
\text { servicios para el } \\
\text { alumno (ocio, } \\
\text { fisioterapeuta, } \\
\text { logopeda..) dentro } \\
\text { de la escuela. }\end{array}$ & $\begin{array}{l}\text { Apoyo centrado } \\
\text { en la escuela: } \\
\text { reutilizando los } \\
\text { recursos y apoyos } \\
\text { humanos, } \\
\text { materiales y } \\
\text { financieros del } \\
\text { propio centro, } \\
\text { creando redes } \\
\text { naturales de } \\
\text { apoyo. }\end{array}$ & $\begin{array}{l}\text { Apoyo externo al } \\
\text { centro, pero en el } \\
\text { que los } \\
\text { especialistas se } \\
\text { comporten como } \\
\text { un miembro más } \\
\text { del centro, } \\
\text { conviviendo para } \\
\text { ayudar a } \\
\text { promover } \\
\text { procesos de } \\
\text { mejora. }\end{array}$ & $\begin{array}{l}\text { Ajuste de la } \\
\text { programación del } \\
\text { aula para crear las } \\
\text { condiciones } \\
\text { necesarias que } \\
\text { permitan la } \\
\text { integración de } \\
\text { todos los } \\
\text { alumnos. }\end{array}$ & $\begin{array}{l}\text { Estructuras } \\
\text { organizativas } \\
\text { sistémicas. }\end{array}$ \\
\hline $\begin{array}{l}\text { Grupos } \\
\text { colaborativos de } \\
\text { profesores para la } \\
\text { resolución de } \\
\text { problemática } \\
\text { escolar. }\end{array}$ & $\begin{array}{l}\text { Establecimiento } \\
\text { de trabajo } \\
\text { colaborativo } \\
\text { entre profesores, } \\
\text { alumnos y } \\
\text { padres. }\end{array}$ & $\begin{array}{l}\text { Colaboración } \\
\text { entre el } \\
\text { profesorado. }\end{array}$ & $\begin{array}{l}\text { Apoyo dentro del } \\
\text { aula mediado por } \\
\text { los profesores. }\end{array}$ & $\begin{array}{l}\text { Establecimiento } \\
\text { de redes de } \\
\text { apoyo y } \\
\text { colaboración } \\
\text { (profesionales, } \\
\text { alumnos, } \\
\text { comunidad). }\end{array}$ \\
\hline
\end{tabular}


Los obstáculos y la complejidad de la educación inclusiva me llevan a persistir en la idea de caminar por aquella vía de construcción del conocimiento que enfatiza el carácter crítico y dialógico del mismo, su fundamentación en la "conversación" y discusión entre teoría y práctica, y su apoyo en la construcción -compartida- de discursos y profesionales. Esto supone superar las ideas simplistas sobre la colaboración (como mero trabajo en grupo), y avanzar desde la colaboración entre iguales hacia la colaboración entre profesionales y agencias diversas, con distinto estatus profesional y adscripción profesional (Parrilla, 2007, p. 35)

¿Cómo se llega a colaborar? Para que la colaboración se pueda llevar a cabo deben confluir una serie de requisitos. En primer lugar, los profesores necesitan tiempo y espacios para colaborar y buscar nuevos modos de hacer escuela, pero también debe existir un clima de confianza para abordar los problemas que surjan sin que nadie se sienta juzgado. ¿Existe ese tiempo? ¿Cuántas veces se reúnen los profesores de un curso para intercambiar métodos de trabajo con los alumnos? ¿Los profesores conocen el trabajo que realizan unos y otros? ¿El profesorado se siente cómodo para poder discutir problemas en su centro escolar? Éstas serían algunas de las cuestiones que servirían de punto de partida para reflexionar sobre la existencia y desarrollo de procesos de colaboración en un centro educativo.

En este sentido, Peters, Johnstone y Ferguson (2005) señalan, a partir del análisis de diferentes documentos elaborados por organizaciones de personas con discapacidad, que entre las variables más importantes para avanzar hacia una educación inclusiva es necesario un nuevo rol del profesor como un modelo de apoyo colaborativo para la resolución de problemas de forma creativa.

De la misma forma, Novoa (2007) considera que hay una condición crítica que depende del equipo directivo, en la medida que es éste quien debe apoyar las iniciativas de los profesores, es decir, debe impulsar o al menos, (nos atrevemos a decir), no impedir la colaboración entre los docentes. Por tanto, será esencial, además del compromiso y la revisión del sistema de creencias de los profesores, el apoyo del equipo directivo a las iniciativas del profesorado, así como estructuras organizativas que sean capaces de soportar los cambios y las continuas mejoras (Kugelmass, 2001).

\section{Algunas barreras que dificultan la colaboración}

Pese a la importancia de la colaboración entre los profesores, claramente documentada durante la pasada década, aún una de las principales barreras que impiden la colaboración reside en los roles rígidos e inamovibles de los docentes, propios de estructuras características de las "burocracias profesionales" descritas por Skrtic (1991).

Uno de estos roles rígidos a los que se hace referencia es al apoyo educativo especialmente referido a los alumnos con Necesidades Educativas Especiales (NEE). Jiménez Trens, Díaz Allué y Carballo (2005) realizaron un estudio en La Rioja con 1.257 profesores pertenecientes a la etapa de Secundaria Obligatoria que describía la valoración del profesorado respecto a su formación psicopedagógica para atender a la diversidad. Los resultados mostraron que en torno al $60 \%$ de los docentes reclamaban más formación en estrategias de enseñanza-aprendizaje para adaptar la enseñanza común a las diferencias individuales. 
Nuestras organizaciones mantienen los roles y estatus diferenciados entre profesores identificados en los centros como especialistas (como profesores de apoyo, logopedas, profesores de compensatoria) y profesores del aula, lo que limita el tipo de colaboración que se puede establecer para responder a las necesidades educativas específicas de los alumnos. Analicemos, por ejemplo, la situación de la relación entre el profesor del aula/ tutor y la figura del profesor de educación especial; desde nuestro punto de vista, existen dos aspectos que condicionan y limitan la relación entre estos dos profesionales: por un lado, las funciones tan difusas del profesor de apoyo en la actualidad y, por otro, la falta de responsabilidad por parte del resto del profesorado para enseñar a los alumnos con discapacidad.

\section{a) Funciones del profesor de apoyo}

Uno de los principales problemas de esta figura profesional es la falta de claridad en sus funciones (Moriña y Puente, 2000). En muchos casos las tareas que se le encomiendan se circunscriben principalmente a ofrecer apoyo educativo a alumnos con Necesidades Educativas Especiales. Además, tal y como exponen algunos estudios, este apoyo se realiza fuera del grupo de referencia. El trabajo realizado por Díez (1999) en León durante dos cursos escolares (1998-1999) puso de manifiesto que en la mayor parte de los casos (el $86 \%$ del tiempo) los alumnos con N.E.E recibían apoyo fuera del grupoaula. Asimismo, otro estudio realizado por Montiel (2002) en Institutos de Educación Secundaria de la Comunidad de Madrid mostró que el $80 \%$ del profesorado de apoyo realizaba su trabajo con los alumnos fuera del aula de referencia. En el mismo sentido, en el estudio Echeita (2004) se señala que un alto porcentaje de los profesores de todas las etapas educativas $(68,5 \%)$ prefieren que la enseñanza de los alumnos con necesidades educativas especiales se realice de forma individual o en pequeño grupo fuera de clase por el maestro de apoyo.

Entre las funciones que tiene que desempeñar legalmente el profesor de apoyo se encuentra la de elaborar junto con el profesorado las Adaptaciones Curriculares Individuales, colaborar con el tutor y el profesorado en el seguimiento y evaluación del alumnado, así como diseñar materiales didácticos. No obstante, aparte de estas funciones, también al profesor de apoyo se le encomiendan otras tareas como la de asesorar al profesorado en la prevención, detección y valoración de los alumnos con problemas de aprendizaje y de su integración social. Sin duda, ésta es una de las funciones que guarda más relación con el cambio educativo para construir una escuela para todos.

En cada centro escolar las funciones de esta figura profesional pueden cambiar considerablemente llevando a cabo más funciones de asesoramiento a profesores en aspectos relacionados con el currículo o con materiales didácticos. Hay centros en los que los apoyos se organizan dentro del aula y se establece un tiempo semanal o quincenal para intercambiar información con los profesores y existen centros en los que los profesores tienen un tiempo para elaborar material didáctico adaptado que está accesible a todos los docentes. También en algunas escuelas el profesor de apoyo ofrece refuerzo educativo a alumnos que no tienen la etiqueta de "Necesidades Educativas Especiales", sino a todos los que necesitan una ayuda extra para alcanzar los objetivos curriculares, y hay casos aislados en los que los profesores de apoyo cumplen un papel esencial a la hora de transformar las concepciones e inercias de los centros. 
York-Barr, Sommerness, Duke y Ghere (2005) a partir del estudio realizado concluyen que existen cuatro dimensiones distintas en el desempeño de un profesor de apoyo y, establecen las responsabilidades de estos profesionales, en el marco de la educación inclusiva.

Como podemos observar en la tabla II, las funciones de este profesional tienen una mayor amplitud que la meramente instructiva y avanzan hacia un modelo sistémico y colaborativo en cuanto que abordan la mayoría de sus funciones junto con otros profesionales.

Por tanto, resulta necesario, cuando el propio marco de integración educativa se está revisando (Plan de Reactivación de la educación inclusiva del Ministerio de Educación, Política Social y Deporte), que se empiece también a replantear las propuestas que se están ofreciendo desde la propia formación del profesorado para modificar modelos de trabajo expertos en otros más reflexivos y colaborativos.

Pero, aparte de la falta de claridad de sus funciones, ¿qué otras dificultades se encuentra este profesional? Cuando se les pregunta a los profesores de apoyo sobre este aspecto, éstos perciben falta de apoyo por parte de los tutores. Cole (2005) llevó a cabo una investigación con 59 profesores de apoyo e indagó acerca de la calidad de la enseñanza-aprendizaje, sus funciones, y el grado de satisfacción con su trabajo. Queremos destacar que tan sólo un $29 \%$ de los participantes opinaban que los alumnos estaban recibiendo el nivel de apoyo adecuado. La mayoría (76\%) consideró que necesitaría más apoyo del resto de profesorado para desempeñar correctamente su trabajo.

En definitiva, resulta imposible abarcar todas las necesidades que tienen hoy en día los centros educativos si dejamos que sólo esta figura lo protagonice. Tal y como señala Muntaner (2002), el cambio educativo tendría que basarse en modificar la concepción de los maestros de apoyo:

El profesor de apoyo deja de ser el profesor particular de los alumnos con necesidades educativas especiales que solventa y trata únicamente la problemática relacionada con estos alumnos, para convertirse en un verdadero apoyo a la escuela en su conjunto y a la dinámica creada en cualquiera de sus aulas en las que participan desde la igualdad todos los alumnos, sin excepciones (Muntaner, 2002, p.30).

Contrariamente a la imagen tradicional de los profesores de educación especial que trabajan con un pequeño grupo de estudiantes en una clase pequeña y tranquila, los profesores deben emerger como coordinadores de un grupo de profesores para asesorar el complejo conjunto de estructuras y recursos del entorno dentro del centro. De hecho, la tendencia mayoritaria en este campo siempre ha señalado la necesidad de reconvertir el rol de los profesores de educación especial que tienen una formación específica en algunos países (Alemania, España, Italia, Portugal...) como asesores de atención a la diversidad (Brodin y Lindstrand, 2007).

b) El papel del profesor del aula/tutor

Como mencionamos anteriormente, tradicionalmente los profesores han derivado su responsabilidad hacia el profesor especialista. Para poder hablar de una escuela sin exclusiones, los profesores deben creer que todos los alumnos de su aula son igual de va- 
Tabla 2. Roles y responsabilidades de los profesores de apoyo dentro de una escuela inclusiva (Tomado de York-Barr, Sommerness, Duke y Ghere, 2005)

\begin{tabular}{|c|c|c|c|}
\hline $\begin{array}{l}\text { Desarrollo de } \\
\text { programas } \\
\text { individualizados }\end{array}$ & $\begin{array}{l}\text { Coordinación de la } \\
\text { puesta en marcha } \\
\text { de programas para } \\
\text { todos los } \\
\text { estudiantes }\end{array}$ & $\begin{array}{l}\text { Ofrecer apoyo } \\
\text { educativo a los } \\
\text { estudiantes }\end{array}$ & $\begin{array}{l}\text { Trabajo directo } \\
\text { con el resto de } \\
\text { profesionales } \\
\text { (no docentes) }\end{array}$ \\
\hline $\begin{array}{l}\text { - Desarrollo y } \\
\text { actualización de } \\
\text { Adaptaciones } \\
\text { Curriculares } \\
\text { individualizadas } \\
\text { - Valoración del } \\
\text { rendimiento de } \\
\text { los estudiantes. } \\
\text { - Adaptación de las } \\
\text { pruebas } \\
\text { estandarizadas de } \\
\text { evaluación } \\
\text { estatales o } \\
\text { locales. } \\
\text { - Realización de } \\
\text { horarios } \\
\text { individuales de } \\
\text { los estudiantes. } \\
\text { - Planificación y } \\
\text { organización de } \\
\text { los periodos de } \\
\text { transición entre } \\
\text { las etapas } \\
\text { educativas. } \\
\text { - Coordinación } \\
\text { con otras } \\
\text { agencias } \\
\text { (servicios } \\
\text { sociales, agencias } \\
\text { laborales, } \\
\text { asociaciones ...) } \\
\text { - Comunicación } \\
\text { con los padres. }\end{array}$ & $\begin{array}{l}\text { - Creación de } \\
\text { redes de apoyo } \\
\text { dentro de la } \\
\text { escuelas, } \\
\text { obteniendo } \\
\text { apoyo de la } \\
\text { administración y } \\
\text { del equipo } \\
\text { directivo } \\
\text { - Colaboración con } \\
\text { los equipos } \\
\text { docentes } \\
\text { (proporcionando } \\
\text { información } \\
\text { sobre las } \\
\text { dificultades de } \\
\text { aprendizaje de } \\
\text { sus alumnos, } \\
\text { enseñando } \\
\text { métodos de } \\
\text { aprendizaje } \\
\text { colaborativos } \\
\text { entre los } \\
\text { docentes y otros } \\
\text { profesionales). } \\
\text { - Seguimiento y } \\
\text { difusión de los } \\
\text { resultados del } \\
\text { plan de } \\
\text { actuación. }\end{array}$ & $\begin{array}{l}\text { - Apoyo a alumnos } \\
\text { de forma } \\
\text { individual o en } \\
\text { pequeño grupo } \\
\text { cuando se } \\
\text { considere } \\
\text { necesario. } \\
\text { - Apoyo educativo } \\
\text { en pequeño } \\
\text { grupo dentro del } \\
\text { grupo clase. }\end{array}$ & $\begin{array}{l}\text { - Intercambio de } \\
\text { información de } \\
\text { los técnicos } \\
\text { educativos, } \\
\text { conserjes... para } \\
\text { establecer los } \\
\text { cambios } \\
\text { necesarios en el } \\
\text { horario del } \\
\text { alumno. } \\
\text { - Entrenamiento al } \\
\text { resto de } \\
\text { profesionales del } \\
\text { centro en } \\
\text { modificación de } \\
\text { conducta, rutinas } \\
\text { relacionadas con } \\
\text { qué hacer en } \\
\text { momentos } \\
\text { críticos, etc.) } \\
\text { - Supervisión de } \\
\text { las actividades } \\
\text { que realizan los } \\
\text { alumnos en el } \\
\text { recreo y en } \\
\text { tiempos de } \\
\text { comida. }\end{array}$ \\
\hline
\end{tabular}


liosos y que todos ellos están en el lugar que tienen que estar. No obstante, esta creencia de la que hablamos existe en función de los valores que en palabras de Booth (2006) cada persona "pone en acción".

Aparte de esta responsabilidad individual, no podemos obviar que el cambio en las concepciones del profesor y, en consecuencia, en las acciones que éste lleva a cabo, está determinado, en gran medida, por la "posición" de la comunidad educativa en el que desarrolla su actividad en relación con la inclusión. Dependiendo de cómo construya el centro en su conjunto su visión de inclusión se pondrán en marcha medidas organizativas, metodológicas para hacer frente a la diversidad pero también, en este proceso es importante la formación del profesorado.

\section{Reflexiones sobre la formación del profesorado}

Los profesores pueden trabajar de forma individual o colectivamente en el diseño y desarrollo del currículo. La forma de trabajo que adopten dependerá de numerosos factores entre los que destaca la cultura escolar en la que se encuentran inmersos. Debemos reconocer que la formación que ha recibido el profesorado (tanto inicial como permanente) en la mayoría de las ocasiones no le ha preparado suficientemente para enfrentarse a situaciones que se encuentran en las aulas y en un contexto que, como hemos mencionado, le está haciendo continuas demandas y planteando constantes retos. La colaboración como cultura profesional constituye un reto ineludible al que la formación del profesorado debe responder.

¿Hasta qué punto la formación inicial del profesorado está incidiendo en sus propias concepciones y le proporciona herramientas conceptuales y metodológicas adecuadas para responder a la diversidad?

Como señalan Gallego y Rodríguez (2007) la formación del profesorado en la atención a la diversidad ha sido categorial, es decir, en función de las deficiencias específicas. Por tanto, se ha potenciado la idea de que la educación especial posee una metodología especializada de cada una de las discapacidades. No obstante, educar para construir una escuela para todos, es asumir la equidad como valor que permite la mejora de la sociedad en la que cabemos todos, es pensar que las diferencias son igual de importantes y valiosas. Y es rechazar la segregación de personas y colectivos. Pero no se trata de un camino fácil y exento de conflictos. El avance hacia una educación inclusiva implica la superación de barreras en ámbitos y contextos diversos, para lo que son necesarias nuevas competencias.

Según Echeita (2006), las competencias profesionales fundamentales que debe tener el profesorado para hacer frente a un desempeño profesional complejo como el que se deriva del discurso de la inclusión educativa, podrían resumirse en las once siguientes:

1. Tener una actitud reflexiva y crítica, propia de un intelectual comprometido, tanto frente a sus propios pensamientos, emociones y prácticas, como frente a la realidad educativa y social.

2. Tener curiosidad e iniciativa para indagar y resolver sus dudas e incertidumbres.

3. Saber buscar y recopilar información relevante sobre las dificultades o problemas con los que se encuentre. 


\section{LA COLABORACIÓN Y LA FORMACIÓN DEL PROFESORADO COMO FACTORES}

FUNDAMENTALES PARA PROMOVER UNA EDUCACIÓN SIN EXCLUSIONES

4. Ser estratégico, esto es, capaz de aplicar procedimientos sistemáticos y ordenados para analizar la información y tomar decisiones.

5. Ser capaz de trabajar colaborativa y cooperativamente con otros, sean compañeros, otro profesional del centro o con las familias en el análisis de la propia realidad educativa y en la planificación, el desarrollo y la evaluación de las iniciativas de mejora que se acuerden.

6. Saber mantener buenas pautas de comunicación, de diálogo y de escucha.

7. Saber pedir y ofrecer ayuda.

8. Mostrar empatía ante las necesidades y emociones de los otros.

9. Saber compartir y entablar relaciones de reciprocidad y de confianza con los compañeros y alumnos.

10. Asumir riesgos y estar abiertos al cambio.

11. Saber fijarse metas para superarse y querer seguir aprendiendo.

(Echeita, 2006, pp. 144-145)

Muchos de los aspectos mencionados al hablar de formación inicial del profesorado, coinciden con las reflexiones realizadas en torno a la formación permanente de estos profesionales. Así, por ejemplo Oliver (2003), considera que esta formación debe contemplar dos dimensiones. Por un lado, una dimensión vertical en cuanto al objeto de la formación, cuyo objetivo principal debe ser la adquisición de aprendizajes que promuevan el desarrollo profesional y la revisión de la práctica educativa con la finalidad de mejorar ésta. También se contempla una dimensión horizontal respecto al sujeto de la formación en donde la reflexión se constituye como una pieza clave. Los procesos de formación continua deben planificarse de forma que el profesorado los entienda como un medio de contraste y revisión de su tarea docente, pero esta reflexión no debe realizarse únicamente de forma individual, sino también de forma conjunta, debe considerar sus necesidades desde la realidad de sus centros, de sus equipos docentes y de sus alumnos.

Atendiendo a las consideraciones anteriores Oliver (2003), propone una modalidad de formación denominada "formación en la práctica" y, por su parte, Jiménez Trens (2007) plantea que el antiguo modelo de impartición de seminarios a los maestros tiene que dar paso a unas comunidades de aprendizaje profesional, vibrantes y constantemente activas.

Como hemos tratado de mostrar con estas reflexiones, no podemos pensar en ningún proceso de innovación y mejora de la educación sin considerar, desde una reflexión compartida, las dificultades y necesidades que tiene el profesorado que se enfrenta día a día a la aventura de enseñar. La colaboración constituye un factor indispensable para aumentar la confianza e innovación. En palabras de Hargreaves (1996):

"La colaboración estimula la asunción de riesgos, una mayor diversidad de estrategias docentes y una sensación de mayor eficacia entre los profesores dado que los estímulos positivos y la retroalimentación sobre las consecuencias de las acciones fortalecen su confianza en sí mismos. Todas estas cosas influyen sobre el aprendizaje de los alumnos y lo facilitan." (Hargreaves, 1996, p. 269). 


\section{Referencias bibliográficas}

Ainscow, M. (1995). Necesidades especiales en el aula. Guía para la formación del profesorado. Madrid: Narcea. [V.O.: Special needs in the classroom: a teacher education guide. UNESCO, 1994].

Arnaiz, P. (2003). Educación inclusiva: una escuela para todos. Málaga: Aljibe.

Booth, T. (2006). Manteniendo el futuro con vida; convirtiendo los valores de la inclusión en acciones. En M.A. Verdugo \& F.B. Jordán de Urríes (Coords.), Rompiendo inercias. Claves para avanzar. VI Jornadas Científicas de Investigación sobre Personas con Discapacidad (pp. 211-217). Salamanca: Amarú.

Brodin, J. y Lindstrand, P. (2007). Perspectives of a school for all. International Journal of Inclusive Education, 11(2), 133-145.

Cole, B. (2005) Mission impossible? Special Education Needs, inclusion and the re-conceptualization of the role of the SENCO in England and in Wales? European Journal for Special Needs Education, 20(3), 287-307.

Díez, E. J. (1999). Integración /desintegración. Organización y Gestión educativa, 5, 3-8.

Echeita, G. (2004). La situación educativa del alumnado con NEE asociadas a discapacidad en la CAM. Psicología Educativa vol. 10, 1, pp. 19-44.

Echeita, G. (2006). Educación para la inclusión o educación sin exclusiones Madrid: Narcea.

Gallego, J.L. y Rodríguez, A. (2007). Tendencias en la formación inicial del profesorado en educación especial. Revista Electrónica Iberoamericana sobre Calidad, Eficacia y Cambio en Educación, 5(3), 102-117.

Hargreaves, A. (1996). Profesorado, cultura y postmodernidad. Madrid: Morata

Jiménez Trens, $M^{\mathbf{a}}$ Asunción (2007). Propuestas curriculares para responder a la diversidad del alumnado de primaria desde contextos inclusivos. Contextos educativos, 10, 29-44.

Jiménez Trens; J.; Díaz Allué, Maㅡ.; Carballo, R. (2006): Respuesta educativa a la diversidad desde la perspectiva del profesorado de la ESO: Estudio en la Comunidad Autónoma de la Rioja. Contextos educativos, 8-9, 33-50.

Kugelmass, J.W. (2001). Collaboration and compromise in creating and sustaining an inclusive school. International Journal of Inclusive Education, 5(4), 47-65.

Martín, E. y Cervi, J. (2006). Modelos de formación docente para el cambio de concepciones en los profesores En J.I. Pozo, N. Scheuer, M.P. Pérez Echevarría, M. Mateos, E. Martín y M. de la Cruz (Eds.), Nuevas formas de pensar la enseñanza y el aprendizaje. Concepciones de profesores y alumnos (pp. 420-434). Barcelona: Graó.

Montiel, L. (2002) Análisis de la puesta en marcha del programa de integración en los centros públicos de educación secundaria de Madrid. El papel del departamento de orientación en su desarrollo. Madrid: Universidad Autónoma de Madrid. Tesis doctoral inédita.

Moriña, A. y Parrilla, A. (2005). Criterios para la formación permanente del profesorado en el marco de la educación inclusiva. Revista de Educación, 339, 517-539. 
Moriña, A., y Puente, D. (2000). La atención a la diversidad en contextos de apoyo. Aula abierta, 75, 135-148.

Muntaner, J. (2002). De la integración a una educación para todos. En R. Marchena y J.D. Martín (Coord). Un modelo de apoyo para una educación de la diversidad (pp. 17-38). Madrid: CEPE.

Novoa, A. (2007). El profesor hoy. La persona, la coparticipación y la prudencia. Cuadernos de Pedagogía, 374, 21-25.

Oliver, M.C. (2003). Estrategias didácticas y organizativas ante la diversidad. Barcelona: Octaedro.

Parrilla, A. (1999). Más allá del conocimiento intelectual sobre la diversidad. Revista de Currículum y Formación del Profesorado, 3(2), 39-55.

Parrilla, A. (2007). Inclusive education in Spain: a view from inside. En L. Barton y F. Armstrong (Eds.), Policy, experience and change: Cross cultural reflections on inclusive education (pp. 19-36). Dordrecht: Springer.

Parrilla, A. y Gallego, C. (1998). Grupos de apoyo entre profesores. Cuadernos de Pedagogía, 270, 29-31.

Peters, S., Johnstone, C. y Ferguson, P. (2005). A Disability Rights in Education Model for evaluating inclusive education. International Journal of Inclusive Education, 9(2), 139-160.

Porter, G. L. (1997). Critical elements for inclusive schools. En Pijl, S.J., Meijer, C.J.W., y Hegarty, S. (Eds) Inclusive Education a global agenda. London: Routeledge Publising

Skrtic, T.M. (1991) Students with special educational needs: Artefacts of the traditional curriculum En M. Ainscow (Ed.), Effective Schools For All (pp. 20-42) Londres: Fulton

York-Barr, J., Sommerness, J., Duke, K. y Ghere, G. (2005). Special educators in inclusive education programmes: reframing their work as teacher leadership. International Journal of Inclusive Education, 9(2), 193-215. 